\title{
The Role of Neutrophils in the Interaction with Leishmania: Far beyond a Simple Trojan Horse?
}

\author{
João Carlos Araujo Carreira, Alba Valéria Machado da Silva \\ SPTI/INERU/IOC, FIOCRUZ, Rio de Janeiro, Brazil \\ Email: jcacarreira@gmail.com
}

How to cite this paper: Carreira, J.C.A. and da Silva, A.V.M. (2021) The Role of Neutrophils in the Interaction with Leishmania: Far beyond a Simple Trojan Horse?. Open Journal of Animal Sciences, 11, 399-421. https://doi.org/10.4236/ojas.2021.113029

Received: February 18, 2021

Accepted: June 22, 2021

Published: June 25, 2021

Copyright ( 2021 by author(s) and Scientific Research Publishing Inc. This work is licensed under the Creative Commons Attribution International License (CC BY 4.0).

http://creativecommons.org/licenses/by/4.0/

\section{(c) (i) Open Access}

\begin{abstract}
Neutrophils are the most numerous leukocyte in mammals and normally they are the first phagocyte observed in recently damaged or infected tissues. They play a key role in the innate immune responses to Leishmania and several other microorganisms, nonetheless an exacerbated neutrophils activity can generate a harmful response to the host, therefore its turnover rate is very important to maintain the homeostasis and averts the host tissue damage. Both apoptosis followed by phagocytosis by mononuclear phagocytes (eferocytosis) and reverse transmigration have been considered the main processes for the clearance of neutrophils from injured or infected tissues. However, the interaction with Leishmania and other microbes, as well as molecules produced by arthropod vectors such as sandflies saliva can modify the behavior of neutrophils, causing immediate lysis to prolong their life. In fact, as a result of a long course of coevolution, several microorganisms have developed skills to avoid neutrophil effector mechanisms and take advantage of neutrophil clearance pathways to promote their spread in the host's body. Leishmania, Chlamydia pneumoniae and Yersinia pestis for example use the efferocytic Trojan horse process for their dissemination and immune protection, in a different way vaccinia Ankara virus and Toxoplasma gondii exploit the neutrophil reverse transmigration for the same reason. Here we present an overview of some characteristics of neutrophils and their different destinations after interaction with several microorganisms, with an emphasis on Leishmania species. It was also suggested the probable role of neutrophils reverse transmigration as another possible route for the spreading of Leishmania in the visceral leishmaniasis.
\end{abstract}

\section{Keywords}

Neutrophils, Leishmania, Reverse Transmigration 


\section{Introduction}

Several species of the parasitic protozoa of the genus Leishmania are the causative agent of leishmaniasis. They are capable of infecting various mammalian hosts, including canids, felids, rodents, marsupials, and humans, being transmitted by the bite of female phlebotomine sand flies of the genera Phlebotomus in Africa, Asia and Europe, and Lutzomyia in the Americas.

Leishmaniasis is considered a neglected disease, affecting over 150 million people worldwide with 350 million at risk in about 98 countries or territories and an incidence of over 2 million new cases per year, estimating 0.5 million cases of visceral leishmaniasis and 1.5 million of cutaneous leishmaniasis [1].

It consists of a spectrum of clinical forms including cutaneous, muco-cutaneous, and visceral forms.

In the Old World, the cutaneous leishmaniasis (CL) mostly tends to spontaneous cure of skin ulcers and long-term immunity to reinfection. Nevertheless, in the New World the disease can produce serious diffuse or muco-cutaneous forms.

The diffuse forms are associated with anergic patients with a deficient cell-mediated immunity that enables the parasite to disseminate in the subcutaneous tissues, spreading from the primary lesion, to multiple areas of the skin forming plaques, ulcers, and nodules over the entire body, resembling lepromatous leprosy.

The muco-cutaneous forms can lead to partial or total destruction of the mucosal epithelia of the mouth, nose, throat, and associated tissues.

Visceral leishmaniasis, also known as kala-azar, is a long-term illness characterized by parasite burdens in the spleen, liver, and bone marrow causing irregular bouts of fever, anaemia, substantial weight loss, inflammation of the spleen and liver.

Complication of visceral leishmaniasis can also cause death or post-kala-azar dermal leishmaniasis that is characterized by a hypopigmented macular, maculopapular, and nodular rash usually in patients who had supposedly recovered from the disease [1].

\section{The Life Cycle of Leishmania}

Classically, the life cycle of Leishmania firstly involves the development of the parasite in the midgut of sand flies, where protozoa, after multiplication phases by binary fission, change from avirulent to virulent metacyclic promastigotes in a differentiation process called metacyclogenesis.

Then, the metacyclic forms migrate to the insect's proboscis, through where they are transmitted to mammalian hosts during the next blood meal, being regurgitated together with saliva and some parasite molecules in a pool of blood in the lacerated dermis.

Once inside the new host, metacyclic promastigotes are engulfed by phagocytic cells where they may change the morphology to amastigotes, which are 
rounded forms with a very short flagellum, only identifiable under electron microscopy.

Amastigotes are specially adapted to survive and multiply within the phagocytic cells, where they can also spread to the different tissues of the host.

Tissue injuries caused by the mechanical action of the sand fly proboscis, in addition to saliva and the presence of the parasite, induce the recruitment of immune system cells to the inoculation site.

The neutrophils also known as polymorphonuclear leukocytes are the first cell to arrive at the site of infection after few hours, followed by inflammatory monocytes, tissue macrophages and dermal dendritic cells that arrive soon after for composing a cast of professional phagocytes.

The parasites phagocytized by the incoming neutrophils, may be either killed or survive inside those cells, even when neutrophils undergo apoptosis.

Apoptotic neutrophils parasitized, when phagocytosed by macrophages allow the transfer of viable leishmanias from one cell to another, in a characteristic way in which the parasite invades the macrophages and, therefore, it remains alive for upcoming multiplication inside of the new cells.

This event has been known as "Trojan horse", for the reason that it favors the silent invasion and survival of Leishmania inside the target host cells, where the parasite can promptly multiply.

The life cycle progresses when protozoa infect macrophages or dendritic cells in the skin and currently, it is assumed that these cells are primarily responsible for the spread of parasites to lymph nodes and other organs such as the liver, spleen and bone marrow, transporting the parasite and enabling the infection of new phagocytic cells present in these tissues.

To complete the cycle, the parasite ends up returning to the skin at different points, probably transported by phagocytes, where they can infect the insect vectors in subsequent blood meals. Thus, macrophages have been considered the primary resident cell for Leishmania in mammalian hosts [2].

However, in addition to the definitive role of macrophages in the Leishmania life cycle, the growing knowledge about the physiology of neutrophils, as well as their interaction with Leishmania and other different microorganisms, suggests that their role in visceral leishmaniasis may be much more important than just a Trojan horse.

In the present article, we review some molecular and physiological characteristics of neutrophils and the interaction between these cells and various microorganisms, emphasizing the Leishmania. It was also speculated a probable function of neutrophils on the Leishmania dissemination from the skin to other organs such as, lymph nodes, liver, spleen and bone marrow.

\section{The Neutrophils}

Neutrophils are the most numerous and one of the main cell types among leukocytes; in mammals they can be identified by their characteristic segmented nucleus and cytoplasmic granules. Considering, for example, the percentages 
between monocytes and neutrophils in the blood, the differences are enormous, while the former represent only $2 \%$ to $8 \%$, the latter are $40 \%$ to $60 \%$ of the total leukocyte population. Therefore, they are the human blood's major cellular elements of the innate immune system, having an anti-infectious and a pro-inflammatory role [3].

Under homeostatic conditions, $10^{11}$ neutrophils leave the bone marrow every day. To produce and supply such an enormous quantity of cells at a high rate, the bone marrow must be able to perform continuously significant levels of granulopoiesis.

In the bone marrow (Figure 1), neutrophils normally develop over a period of 14 days, when they are released into the blood. In peripheral blood, although initially considered as having a short half-life, being cleared in the liver, spleen and bone marrow, after around 12 to $18 \mathrm{~h}$. Subsequent studies have shown that it can take much longer, almost 3.75 days, and in tissues, it has been estimated at 6 to 15 times longer [4].

Lately, the neutrophils lifespan in blood was defined as around $5.4 \mathrm{~d}$, changing the initial idea of those cells as a short living and generated in enormous quantities merely to kill microbes [3].

More recently, studies involving neutrophils have been shown that those cells have a multifunctional role acting as in the innate as well as in the adaptive immune response, many times making a bridge between then [5] [6].

\subsection{Neutrophil Surface Molecules}

Neutrophils have a large number of tools represented by surface molecules used to progress from bone marrow to blood, eventually being able to migrate into tissues in cases of injury or complete their life cycle, flowing to the liver and spleen or returning to the marrow bone, to be removed by resident macrophages in those places (Figure 2).

Many surface molecules may function as a passport permitting their migration through several natural barriers such as endothelium and extracellular matrix, but also represent phenotypic characteristics of certain subpopulations related to interaction with different cell types and antigens.

\section{Myelopoiesis}

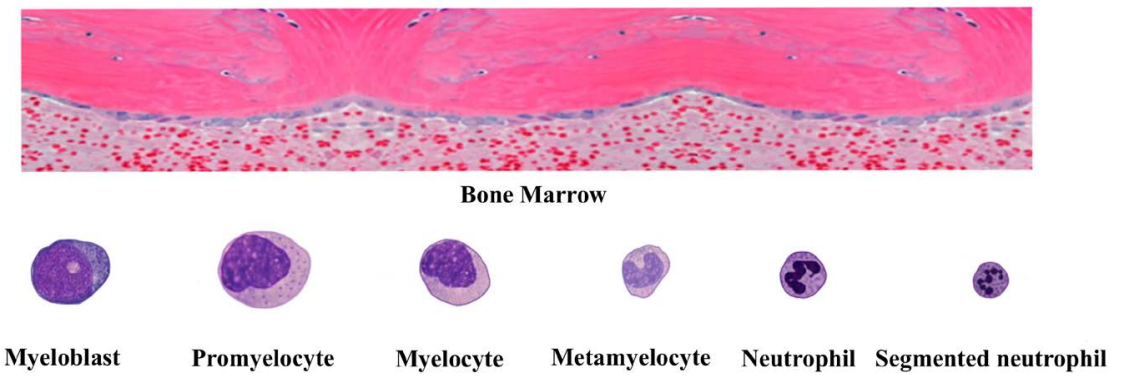

Figure 1. Myelopoiesis-Neutrophil development in the bone marrow. 


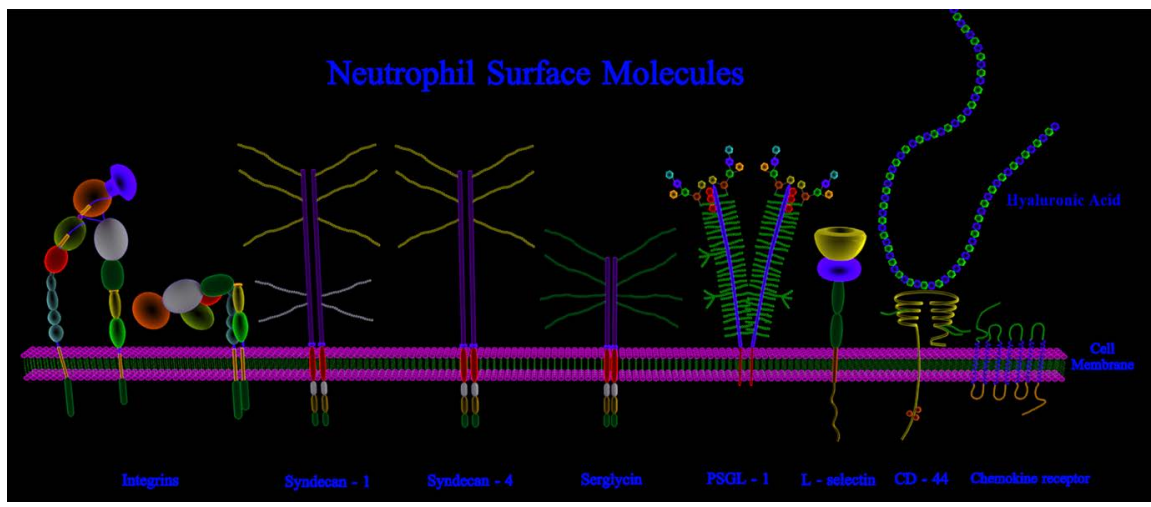

Figure 2. Neutrophil surface molecules-Schematic view of some neutrophil surface molecules related to cell function.

Proteoglycans and hyaluronan comprise the neutrophil surface layer. The integrins are related to, rolling, arrest, adhesion, crawling, transmigration and diapedesis. The CD 44, L-selectin and PSGL-1, respectively the first for tethering, rolling, arrest and adhesion, while the others for tethering and rolling.

CXCR molecules can promote the retention of neutrophils in the bone marrow or the recruitment of these cells to inflammatory foci from the bone marrow.

Neutrophils exhibiting CCR surface molecules are activated and stimulate the amplifying neutrophil recruitment, returning of Ag-loaded neutrophils from dermis to bone marrow, trans-endothelial migration through interaction with monocyte, migrate reversely and to lymph nodes [7].

\subsection{Neutrophils Effector Mechanisms}

In addition to those important tools, the neutrophils also present several "weapons" to destroy invasive microorganisms. They can phagocyte and kill invading microorganisms intracellularly by the formation of phagolysosome composed of anti-microbial molecules like, enzymes and reactive oxygen species (ROS).

Those cells also kill germs extracellularly by the liberation of antimicrobial molecules, stored in their granules through the formation of a structure named neutrophils extracellular traps (NET). In fact, NETs are extracellular, web-like structures comprised of cytosolic and granule proteins that are assembled on a framework mostly composed of histones and DNA, as from the nucleus as well as from the mitochondria in a lesser quantity [8].

In addition, the antimicrobial role, the neutrophils are competent to express genes encoding inflammatory mediators such as growth factors, chemokines and cytokines [3].

Actually, it has been suggested that neutrophils do not comprise just a population of equal innate effector cells and, like lymphoid cells, have subsets with important differences in both phenotype and function [9]. As examples, they have been included, immature and hypersegmented neutrophils as well as low-density granulocytes and granulocyte myeloid-derived suppressor cells. 
To perform their function, these cells are capable to leave the circulation to the tissues, through transendothelial migration, achieving the rolling, adhesion and crawling.

Interestingly, neutrophils can also present diverse ways of death mechanism, like, necroptosis and apoptosis. Those modes of cell death have several effects on the behavior of neighboring cells, like macrophage, for example that through efferocytosis, they phagocytize apoptotic neutrophils and may either disseminate the inflammation or stimulate the resolution [7].

Likewise, neutrophils are also competent to reverse migration, returning to blood and/or lymphatic circulation, after infiltrating other tissues. These reverse-migrating neutrophils, like macrophages, are also able to interact with other immune cells, such as natural killer, B and T lymphocytes, as well as with dendritic cells [7].

\subsection{Neutrophil Reverse Transmigration}

It is known that neutrophils are the first cells to respond to innate immune stimulation, but in many situations tissue damage is followed by the invasion of microorganisms, consequently these cells are also often the first to reach the sites of entry of large numbers of microorganisms, including Leishmania.

Despite the fact that the neutrophil recruitment is vital for protection against microorganisms, the resolution of the inflammation is also decisive, because its retention can cause tissue damage in addition to the chronification of the inflammatory response.

Traditionally, it was established that the control of neutrophil-mediated inflammation in tissues would occur exclusively through a process called efferocytosis, in which necrotic or apoptotic neutrophils are eliminated after being phagocyted by other phagocytic cells like, macrophages and dendritic cells [10].

However, more recently, a reverse transmigration pathway has been fully accepted as a very important mechanism for the neutrophil clearance from the site of inflammation [11].

Regarding the phenomenon of neutrophil reverse transmigration, despite the work of Hughes et al. [12] that clearly demonstrated neutrophils have the ability to leave sites of inflammation and return to the bloodstream. It has been only widely recognized more recently, starting in 2006, by a study in zebrafish showing neutrophils migrating away from a wound and back into the vasculature [10] [13], and in vitro with human neutrophils showing, they undergo a type of bidirectional movement that they termed reverse transmigration (RT) [11].

Later, it was confirmed that mice neutrophils also exhibit (RT) through a junctional adhesion molecule (JAM-C)-dependent pathway and that this could be associated with distant organ inflammation [14].

More recently, Wang et al. [15] showed that although neutrophils were needed for tissue healing in the course of the early stage of inflammation caused by thermal hepatic injury. After $12 \mathrm{~h}$, most of them migrate away from the 
wound place back into the vasculature, while those who remained in place showed low rates of apoptosis, sustaining an alternative process for neutrophil resolution, besides local apoptosis [10].

Consequently, matured neutrophils in response to the wound and infection readily migrate from the bone marrow to the circulation. At this stage, they are activated and can transmigrate through endothelial cells to reach inflamed tissues.

Once at the site of inflammation, neutrophils play their role in the immune system, using the arsenal of weapons to kill invading pathogens. After all, the process of cells clearance starts and several neutrophils are phagocytized after suffering necroptosis or apoptosis.

Nevertheless, some sub-populations of neutrophils are able to get back into the circulation again through reverse trans-endothelial migration (rTEM). Those neutrophils, while in circulation, can respond to a second stimulus or caught in the reticuloendothelial system mostly in the liver and spleen, through macrophage clearance after undergoing apoptotic cells.

Likewise, aged neutrophils after reverse transmigration can return to the bone marrow and interact with different cell types. Thus, neutrophils can modify the expressions of surface markers according to the environment and rTEM can contribute to the fate of their activities [16].

Among the various roles of reverse transmigrated neutrophils in the immune system, they can be included: 1) inhibition of T cell proliferation; 2) stimulation of antibody production; 3) induction of immunoglobulin class change; 4) elimination of excessive neutrophils from the tissues; 5) positive regulation of neutrophil reverse migration from the injury site; 6) production of interleukin 17A (IL-17), a cytokine involved in the development of many autoimmune diseases, as well as in inflammatory conditions and increased production of ROS and NET formation.

Despite the diverse attributes of neutrophils, microorganisms from different groups have been developed strategies to evade the killing by those cells.

\subsection{Mechanisms of Resistance of Leishmania to Neutrophil Killing}

Leishmania, after millions of years of co-evolution with its mammalian hosts, has developed several survival mechanisms, not only to escape the killing of neutrophils, but also to obtain advantages for its dissemination in the host body [17].

Actually, different species Leishmania have been developed in particular ways for circumventing the effector mechanisms of defense of neutrophils. They do that through the blocking the formation of phagolysosomes, inhibiting or resisting the formation of reactive oxygen species (ROS). Some species can survive intracellularly not in the phagolysosomes, but inside of non-lithic compartments with membranes that exhibit characteristics of endoplasmic reticulum.

In addition, the life span of neutrophils can even be modulated during the interaction with different species of Leishmania, causing a delay or acceleration in the process of cell death. Correspondingly, other microorganisms have devel- 
oped similar strategies to survive and obtain advantages during the interaction with neutrophils, favoring the establishment of infections.

\subsubsection{Interference on Phagolysosome Formation}

Through "in vitro" studies, it has been demonstrated that Leishmania major and Leishmania donovani promastigotes regulate granule fusion with phagosomes, allowing azurophil but preventing gelatinase granule fusion with parasite-containing phagosomes [17] (Figure 3). Some bacteria like Leishmania parasite species also interfere on the phagolysosome formation; nevertheless, they differently hinder the fusion of the phagosome with azurophilic granules [8].

As examples of those bacterias we can see, Neisseria gonorrhoeae, Salmonella [18] and Mycobacteria. This last specifically retain Rab 5a and Syntaxin-4 on the phagosome membrane to block the fusion of azurophilic granules in human neutrophils [19], besides promoting the inhibition of pinosome formation [20] [21] [22] [23].

\subsubsection{Interference on Reactive Oxygen Species (ROS) Production and Escape from Neutrophil Phagosomes}

With respect to ROS production in the interplay between Leishmania and neutrophils, the results also depend on the parasite species. L. major does not elicit the

\section{Mechanisms Utilized by Leishmania spp to Survive to Neutrophil Killing}
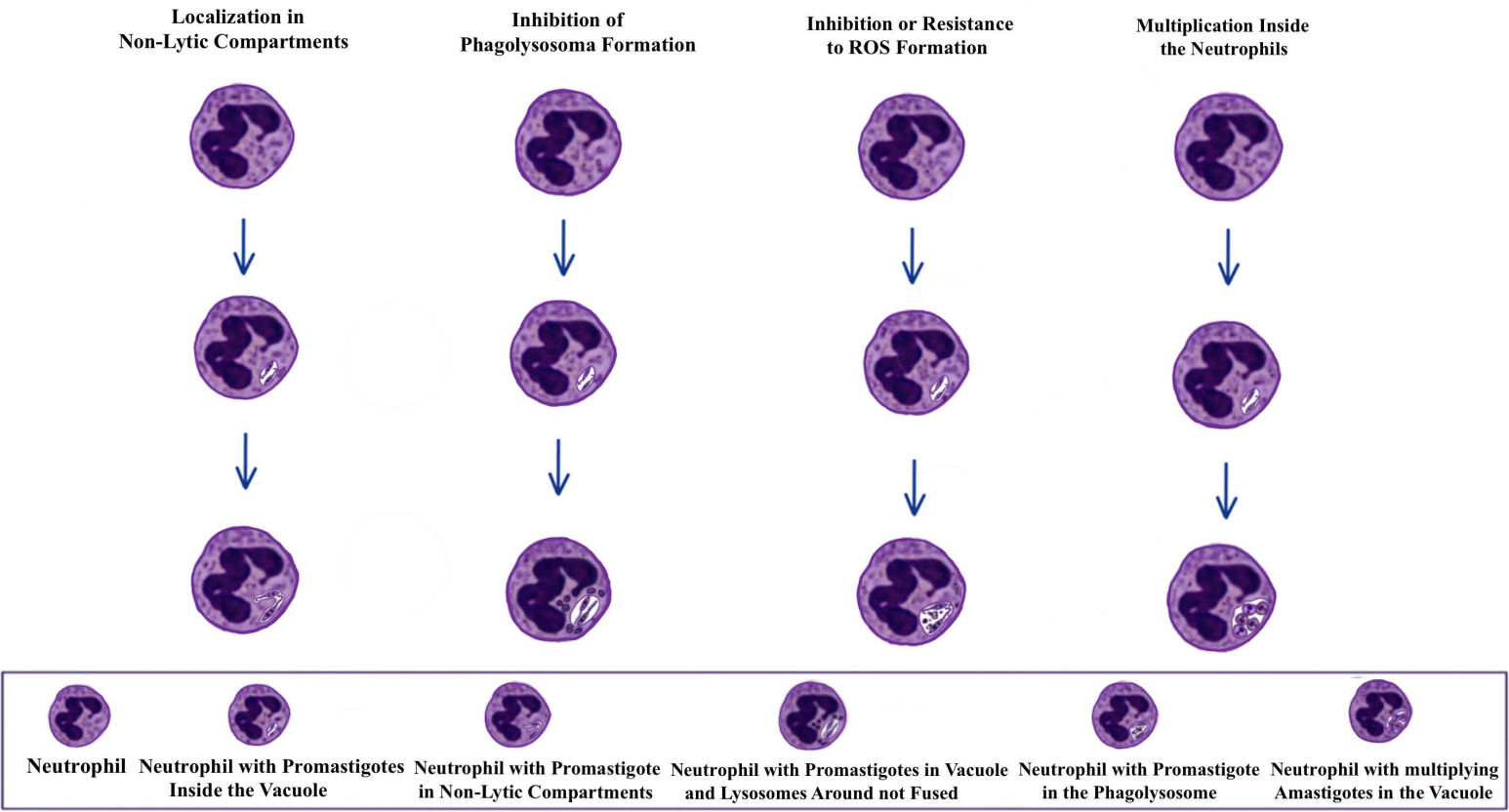

Figure 3. Mechanisms utilized by Leishmania spp. to survive to neutrophil killing-Schematic view of some mechanisms of resistance of Leishmania spp. to neutrophil killing: In the first column, the parasite, after being phagocytized by a neutrophil, escapes from the phagolysosome and is located in non-lytic compartments of the cell. In the second column, the parasite, after being phagocytized by a neutrophil, interferes with the formation of the phagolysosome avoiding the fusion of the lysosomes to the phagocytic vacuoles. The third column shows that Leishmania spp, after being phagocytized by a neutrophil, can inhibit or survive the production of reactive oxygen species (ROS). The fourth column shows that Leishmania spp, after being phagocytized by a neutrophil, can multiply inside the cell. 
generation of ROS after been phagocytized by human neutrophils. L. braziliensis infection induces high levels of ROS of human and murine neutrophils; nevertheless, in human neutrophils it did not affect parasite survival [17] (Figure 3).

Similar to Leishmania, several other microorganisms have been described as affecting the production of ROS in neutrophils, stimulating or inhibiting, but also misdirecting the oxidase complex away from the phagosome.

Bacterial pathogens for example they generate many enzymatic antioxidants to impair the ROS effect and diminish damage caused by oxidative stress. Staphylococcus aureus produces superoxide dismutases that catalyze the dismutation of superoxide to hydrogen peroxide. Then the hydrogen peroxide can be further decomposed to water and oxygen by catalases, like also occurs in Escherichia coli.

Streptococcus pneumoniae and S. pyogenes, can break down the ROS, respectively through enzymes of the thioredoxin and glutathione systems [24] and pathogenic Yersiniae use a type III secretion system (T3SS) translocate effector proteins into the neutrophil cytoplasm to interfere with NADPH oxidase.

Likewise, Pseudomonas aeruginosa type III cytotoxic effector ExoS inhibits ROS production in human neutrophils and, similar to Yersinia, it functions as a GTPase-activating protein. Francisella tularensis and Anaplasma phagocytophilum can control NADPH oxidase assembly by unsettling intracellular signaling. In an analogous way, Chlamydia pneumoniae inhibits PMA- and fMLF-induced oxidative burst by disrupting calcium transients [23] [25] [26].

Anaplasma phagocytophilum inhibits the rescue of flavocytochrome b558 to the phagosomal/vacuolar membrane, Opa-negative Neisseria gonorrhoeae accumulate the same molecule, but exhibit defects in the recruitment of p47phox and p67phox, which reduces the ROS production [26]. Differently, the Helicobacter pylori directs the NADPH oxidase to the outer cell membrane, guiding ROS to the extracellular space [23].

Leishmania donovani was shown to traffic to non-lytic compartments of neutrophils to escape the neutrophil killing [17] (Figure 3). Similarly, Streptococcus pyogenes also are able to escape from neutrophil phagosomes [23].

\subsubsection{Multiplying inside Neutrophils}

It was demonstrated that a strain of $L$. mexicana is able to replicate within neutrophils showing that those cells may function as a shelter for the parasite replication during the chronic phase of infection [17] (Figure 3). Likewise, Anaplasma phagocytophilium, Chlamydia trachomatis, Chlamydia pneumonia, Legionella pneumophila and Yersinia pestis are also able to multiply inside neutrophils [23] [27]. In some of those microorganisms the infected neutrophils may be a route for noninflammatory infection of macrophages in a mechanism firstly described in Leishmania, called as "Trojan Horse" [25].

\subsection{Resistance to NETS}

As in the other neutrophil defense mechanisms concerning to NETs, different 
species of Leishmania can use different strategies to ensure their survival and consequently the progression of the infection.

Leishmania can affect the formation of those structures; in addition of being able to digest them or even resist their toxicity. It was showed that Leishmania infantum prevent NET formation by suppressing or causing a reduction on effectiveness of the oxidative burst (Figure 4).

Another way to evade the NET killing by L. infantum, is the expression of the enzyme 3' nucleotidase/nuclease that degrade the DNA backbone of NETs [17] (Figure 4). L. amazonensis has a large amount of lipophosphoglycan molecules (LPG) forming a thick glycocalyx that, despite the induction of NET, confer resistance to NET-mediated death (Figure 4). In L. donovani, although the LPG has been shown not to induce NET formation, it can also provide protection against these killing structures [17] (Figure 4).

The neutrophil extracellular trap evasion behavior of Leishmania may be comparable to several other microorganisms. Streptococcus from A and B groups, arrange molecules resembling sialic acids that decrease the ROS consequently, reduce lysis by the Net (NETosis). $P$. aeruginosa restrain NETosis by covering themselves with sialylated glycoproteins from host [28] and Bordetella pertussis as well $S$. pyogenes, they also present mechanisms of suppression of NET formation [28].

As in some Leishmania species, other pathogens can also resist NET-mediated killing, for example, Pseudomonas aeruginosa, despite being a potent NETose

Escape Mechanisms used by Leishmania spp to Survive to Neutrophil Extracellular Traps (NET)

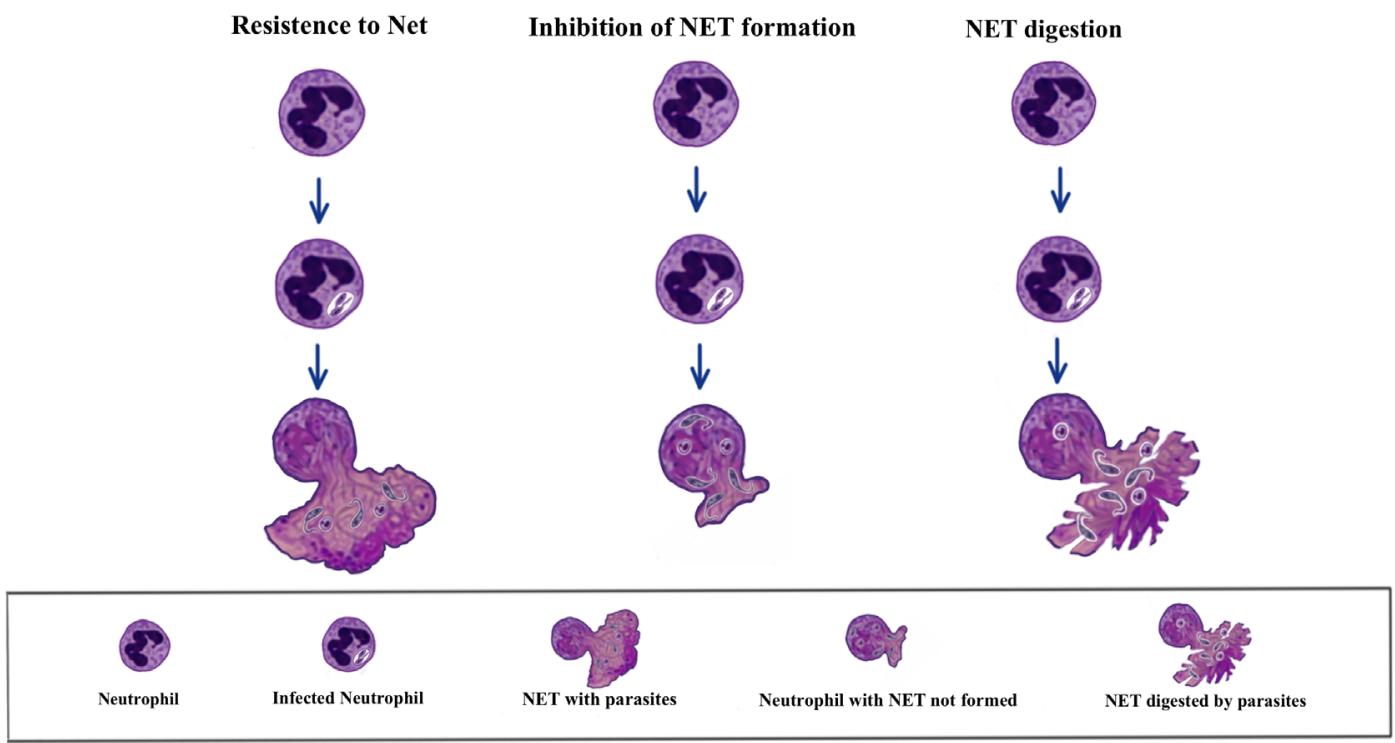

Figure 4. Escape mechanisms used by Leishmania spp. to survive to Neutrophil Extra cellular Traps (NET)-Schematic view of Leishmania spp. escape mechanisms to survive to neutrophil extracellular traps (NETs): In the first column, some Leishmania spp. can survive the NET formation. The second column shows that some Leishmania spp. can inhibit the formation of NET. The third column shows that some Leishmania spp. can promote NET digestion. 
inducer, it has been observed that some clinical isolates are resistant to this mechanism [8].

In cases of primary influenza A infection that causes an increase in the formation of NETs, resistant strains of Streptococcus pneumoniae can use these NETs to multiply and persist at the site of infection. Likewise, Haemophilus influenzae integrates NETs into biofilms, allowing the establishment of infection, but Neisseria gonorrhoeae has a supposedly secreted thermonuclease that promotes NET degradation, preventing the death of the bacteria and allowing infection [29] [30].

\subsection{Modulation of Neutrophil Behavior Induced by the Interaction with Leishmania and other Microorganisms}

As is known, during the first stages of infection by certain microorganisms in the dermis, neutrophils are the first cells to reach the site of infection and in a short time, a large number of these cells arrive attracted by the chemotactic gradients generated around these sites. They phagocytize and degrade cells from damaged tissues, as well as various types of particles, such as parasites and bacteria, which are injected together with the phlebotomine saliva.

Later, during the clearance phase, other phagocytes, such as macrophages and dendritic cells, appear at the site of inflammation to remove apoptotic neutrophils besides remaining particles. In spite of presence of a great number of professional phagocytes, leishmanias and other microorganisms can survive and present similar abilities to and take advantage and ensure the progression of the infection.

After being engulfed by neutrophils, some pathogens can control the behavior of the host cell, slowing down or speeding up the process of apoptosis. Respectively, allowing microbes to persist and multiply within healthy cells or to remain in apoptotic neutrophils to be phagocytized by macrophages and/or dendritic cells subsequently infecting them. In these cases, when phagocytic cells are post-infected after engulfing infected apoptotic neutrophils, this phenomenon has been called as "Trojan horse", due to the silent transfer of the parasite infecting new cells [31].

In certain species of Leishmania, temporary inhibition or delayed apoptosis of neutrophils has been described as allowing the parasite to protect itself within these cells. As can be seen in L. mexicana that does not induce rapid apoptosis of dermal neutrophils in vivo.

On the other hand, in some others, such as L. major and L. braziliensis, for their ability to induce neutrophil apoptosis, or $L$. donovani that causes autophagy in neutrophils derived from human blood and subsequent engulfment by macrophages [32]. It has been suggested that they behave according to the "Trojan Horse" model (Figure 5).

Essentially, the results of the interaction between neutrophils and Leishmania can vary depending not only on the species of the parasite, but also on the origin 


\section{Escape Mechanisms used by Leishmania spp to Survive to Neutrophil Killing}

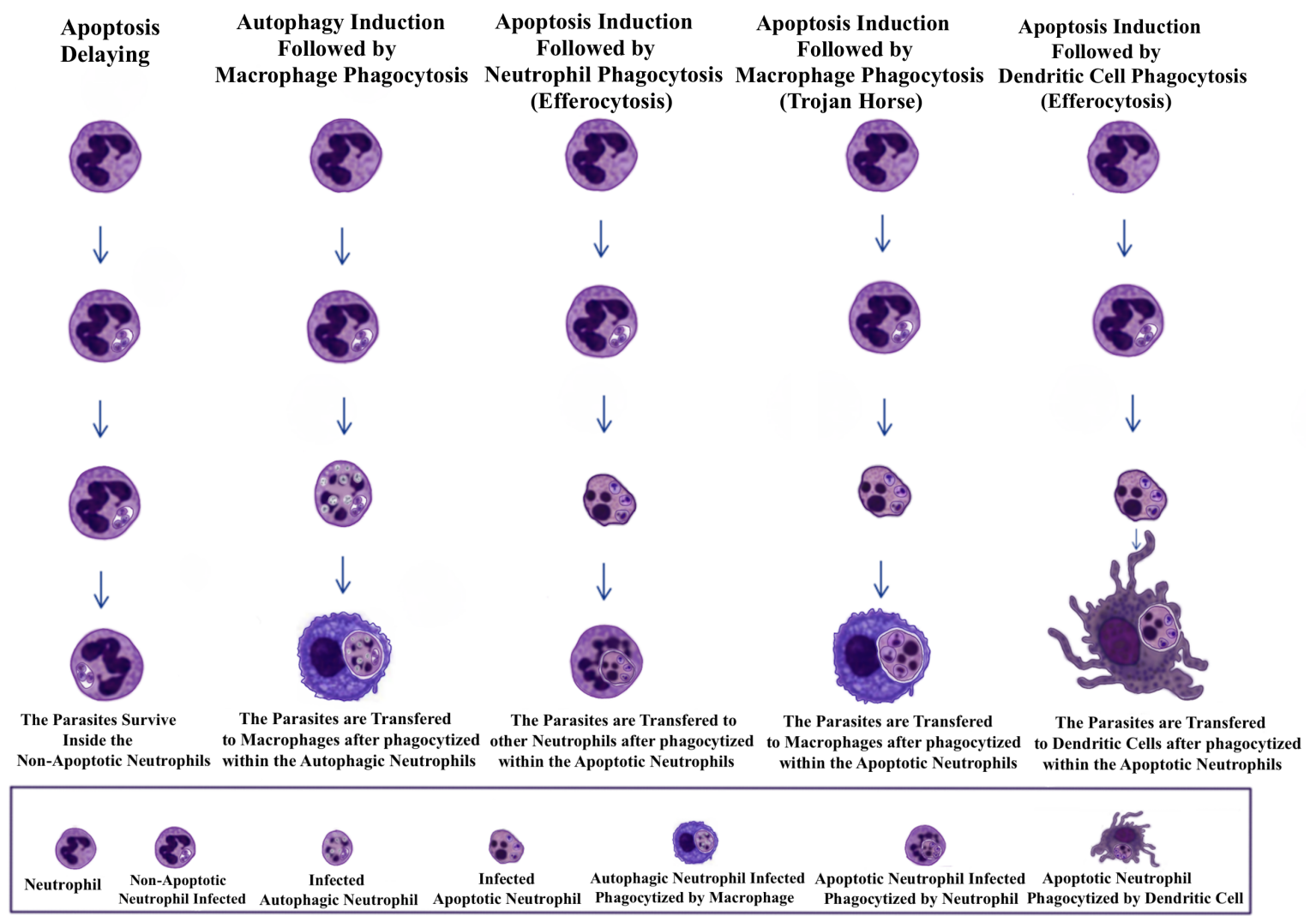

Figure 5. Escape mechanisms used by Leishmania spp. to survive to neutrophil killing-Schematic view of some mechanisms utilized by Leishmania spp. for modulating the behavior of neutrophils to survive to cell killing. In the first column, the parasite, after being phagocytized, induces a delay in the apoptosis of the neutrophil, favoring its survival inside the host cell. In the second column, the parasite, after being phagocytized, induces the autophagy of neutrophils and later phagocytosis by the macrophage, favoring its silent transfer and survival inside the new host cell. In the three remaining columns, the parasite, after being phagocytized, induces apoptosis of neutrophils, also promoting a silent transfer, respectively, to three different new host cells: neutrophils (column 3), macrophages (column 4) and dendritic cells (column 5).

of these host cells [33] [34]. In fact, even when neutrophils are from the same tissue and are infected with just one species of Leishmania, the results may vary.

L. major, for example, has been reported to be able to delay apoptosis in peritoneal induced mouse neutrophils, as well as in human blood-derived neutrophils, but not in those cells derived from mouse dermis [33] (Figure 5). Respectively, after the interaction between $L$. infantum and human neutrophils isolated from venous blood, it was observed that most cells responded to parasite interactions followed by parasites uptake with ROS production and/or activation. Nevertheless, a relative amount of neutrophils did not show answer [35] (Figure $5)$.

These results are in agreement with the concept that neutrophils are effectively composed of several different subpopulations [36]. On the other hand, the variability of the parasite can also influence the destination of the infection, as it is observed with $L$. major, where different strains can trigger different cytokines, as 
well as have different survival rates in neutrophils [37].

In dogs with visceral leishmaniasis, the function of neutrophils is altered according to the stage of the disease. While in the severely affected animals those cells presented very low levels of oxidative burst and increased apoptosis occurred in association with uremia. In moderate stage, there was increased superoxide production without any change in neutrophil viability [38].

Like Leishmania, with regard to the modulation of neutrophil behavior, other microorganisms also exploit similar mechanisms to establish the infection.

For example, Chlamydia pneumoniae and Yersinia pestis have been described as inducing the neutrophil apoptosis also using an efferocytic Trojan horse process for their dissemination and immune protection, by exploiting neutrophils as cell vectors. In the case of $Y$. pestis, it also can replicate within those cells [25] [27] [39] [40]. Several other pathogens can induce neutrophil apoptosis such as Borrelia hermsii, Streptococcus pneumoniae, Mycobacterium tuberculosis, Listeria monocytogenes and Pseudomonas aeruginosa as well as Aspergillus fumigatus hyphae and conidia, besides HIV and Influenza A [24] [26].

Differently, Escherichia coli delays neutrophil apoptosis, increasing the amount of neutrophils during the initial stage of inflammation, consequently creating an opportunity for the clearance of invading pathogens. Several other microorganisms also have the capacity of delaying of neutrophil apoptosis such as, Anaplasma phagocytophilum, Neisseria gonorrhoeae, Francisella tularensis (A and B strains) [26] [27] [41].

In addition, some organisms have been reported to be capable of even inhibiting neutrophil apoptosis, such as Francisella novicida, Histoplasma capsulatum and human cytomegalovirus [26].

\subsection{Reverse Trans-Migrated Neutrophils and Pathogens Dissemination}

Through the long process of coevolution, infectious microorganisms have been developing similar ways to bypass each neutrophil effector mechanism for establishing the infection. As previously mentioned, currently it is well known that the fate of neutrophils after some time of reaching a site of inflammation goes far beyond a cell that is about to die and, subsequently be phagocytosed mainly by macrophages or dendritic cells.

Although the clearance of neutrophils from the tissues may be partially achieved by the efferocytosis mechanism, through their uptake by macrophages after they had become apoptotic. In the course of inflammation, activated neutrophils can extend their survival by developing resistance to apoptosis and an alternative way to remove infiltrated neutrophils from tissues may be proceeded by their reentry into the lymphatic or blood vessels utilizing the trans-endothelial migration [16].

Since the description of the phenomenon of reverse transmigration of neutrophils, it has been shown that pathogens could also benefit in this case, using neutrophils as taxi drivers to transport them from one tissue to another. It was 
also suggested, that reverse-migrated neutrophils could contribute to dissemination of systemic inflammation [42].

Moreover, neutrophils have been shown to leave inflamed or infected sites, travel through lymphatics and relocate to lymph nodes or bone marrow affecting host defense. These neutrophils were able to transport live pathogens to lymph nodes and modulate lymphocyte proliferation [43].

Specifically, neutrophils were able to transport modified vaccinia Ankara virus from the dermis to draining lymph nodes and bone marrow eliciting a $\mathrm{T}$ cells response virus-specific CD8+ but not CD4+. This response was characteristically restricted in the bone marrow and the draining lymph nodes, preceding to blood circulation [44].

Infection with the modified wild-type vaccinia Ankara virus has also been shown to elicit the expression of CCL2, CCL3, CCL4 and CXCL10 by monocytes and these factors may function as chemo-attractive agents for the migration of neutrophils in tissues [44].

In addition, scarce neutrophils were observed in the blood and bone marrow of $\mathrm{CCR} 1^{-/-}$mice, suggesting the importance of this receptor for the migration of neutrophils from infected tissue. Besides, there was non-need of CCR1 for neutrophil access to bone marrow in both neutrophil-depleted and CCR1-/-mice, virus-specific CD 8+ responses were lost [44].

Still considering the possibility of neutrophils transporting pathogens through different tissues, it was proposed that, for some intracellular parasites the presence of neutrophils at the site of infection could be advantageous for their spreading to target cells and/or organs, and could represent an important step for the disease permanency [26].

Additionally, studies involving experimental infection of mice with the parasitic protozoa Toxoplasma gondii combining with two-photon microscopy revealed a trans-epithelial migration of neutrophils. These cells exhibited a significant role on the spread of infection in the small intestine. They behaved like as "motile reservoirs" of $T$. gondii, revealing a retrograde pathway for parasite spread in the intestine.

Thus, neutrophils were able to transport $T$. gondii through biological barriers and remain motile in the mucus-filled intestinal lumen. In addition, the parasite-containing cells themselves were able to migrate through the epithelial cell layer of an uninfected villus and, consequently, creating new points of infection [45]. Neutrophils were also observed transporting latex beads to the bone marrow four hours after intravenous injection [44].

All those above stated information about the most recently better studied phenomenon of neutrophils reverse migration, show two key information that may be relevant to the relationship between these cells and Leishmania: 1) the reverse trans-endothelial migration represents an important physiological process related to the clearance of neutrophils from the site of inflammation. 2) During the reverse migration of neutrophils, they can carry pathogens, from small viral particles to considerably larger organisms, such as protozoa. 
Reverse-migrated neutrophils also exhibited an activated morphology without long-term behavioral alterations, being able to respond as usual to a secondary challenge producing an effective antimicrobial response to Staphylococcus aureus for example [46].

\subsection{The Probable Role of Reverse Trans-Migrated Neutrophils in the Leishmania Dissemination}

Based on all of the above information, including similarities in behavior of leishmanias and various microorganisms interacting with neutrophils, in addition to the recognized importance of neutrophils in Leishmania infections, we are proposing that neutrophils can also play some role in the spread of Leishmania from the skin to other organs or tissues of the host.

In all models that exemplify the Leishmania life cycle, the main ways proposed for the parasite to leave the skin to reach other organs are basically, through transport by macrophages and dendritic cells. Thus, these cells would be the main agents responsible for the spread of the parasite, because after they phagocyte free parasites and/or apoptotic neutrophils containing parasites by efferocytosis (Trojan horse mechanism), they would carry the parasites together into the lymphatic or blood vessels, subsequently reaching other tissues or releasing viable parasites into the vessels after cell lysis.

Here we are suggesting one more likely mechanism for the spreading of Leishmania in the body of the mammalian hosts, where besides dendritic cells and macrophages, the neutrophils could also be able for the transporting of parasites from the skin to other tissues.

Accordingly, the reverse-migrated neutrophils, after phagocytize the Leishmania could access the lymphatics or blood vessels and from the lymphatics, the parasites inside the neutrophils could easily reach the lymph nodes. Alternatively, once in the blood, parasitized neutrophils could be redirected to other sites of inflammation or infection in the skin for example, since they maintain an activated phenotype [47].

Likewise, after some time, when neutrophils have expressed surface receptors indicative of senescence and subsequently when reaching the spleen, liver and bone marrow, they could be cleared being phagocytized by resident macrophages or dendritic cells. As for the neutrophils that fulfill their designated patrolling lifetimes, although the majority will retire in the liver (29\%) and spleen (31\%), many will return to the bone marrow (32\%) for clearance [47] (Figure 6). Thus, the parasites also could be released inside those new tissues to infect other cells after neutrophil lysis.

Several aspects could serve as support for our theory, the undeniable importance of neutrophils on the interaction between Leishmania and the mammal hosts defenses, have been already fully proven through "in vivo" and "in vitro" studies involving several different species and strains of Leishmania.

Those studies included, infection by sand fly bite or needle injection, different routes of infection (intravenous, intradermal, subcutaneous or intraperitoneal) 
HIPOTHETICAL LIFE CYCLE OF Leishmania Spp CONSIDERING THE NEUTROPHIL REVERSE TRANSMIGRATION AS A MECHANISM OF PARASITE DISSEMINATION

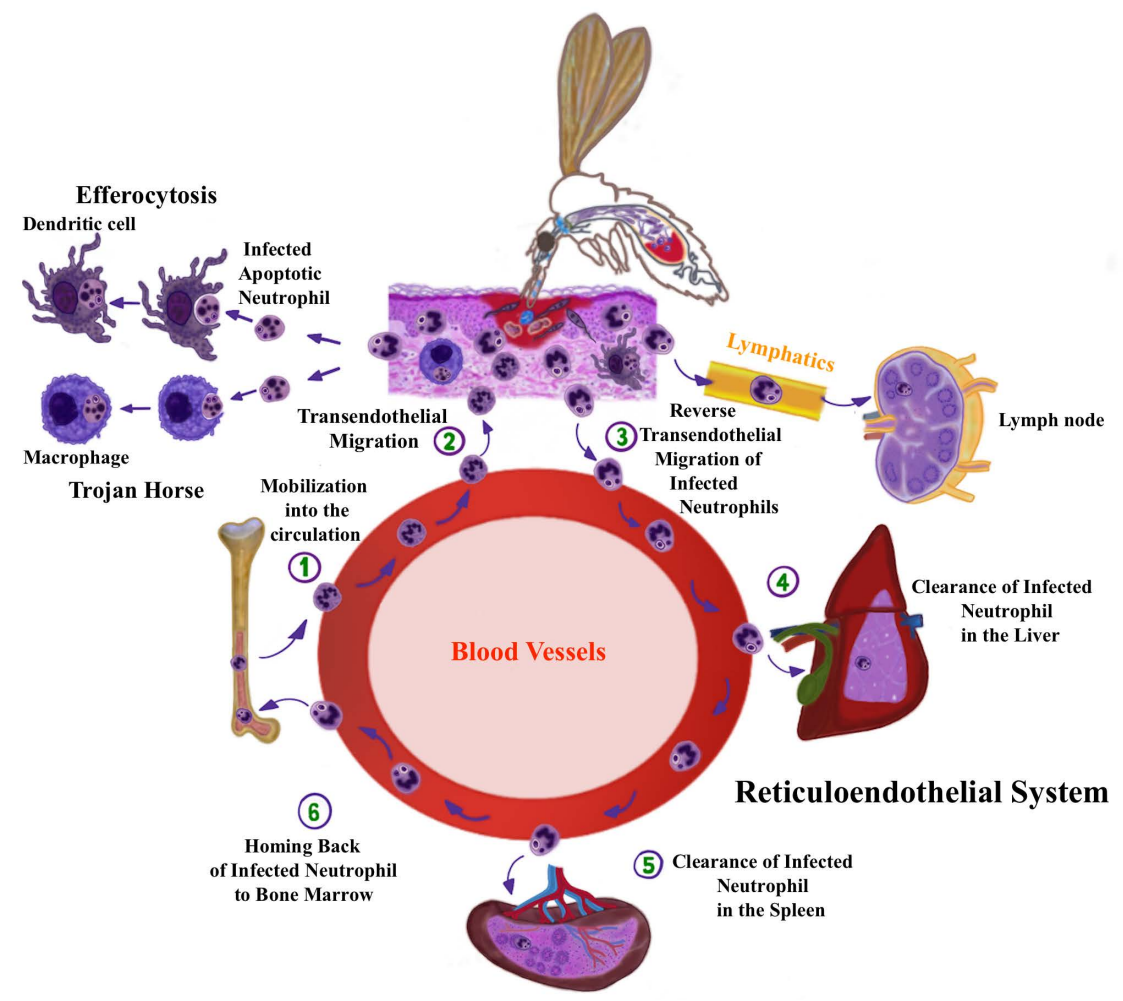

Figure 6. Hypothetical life cycle of Leishmania spp. considering the neutrophil reverse transmigration as a mechanism of parasite dissemination-The life cycle of visceral leishmaniasis considering neutrophils as one of the cells involved in the spread of the parasite in mammal hosts: 1) Neutrophils from bone marrow access the bloodstream. 2) Due to the chemotactic factors produced by tissue damage, and by the presence of the phlebotomine saliva, the parasites as well as some factors produced by them. Neutrophils transmigrate from the bloodstream to the injured area. Once in the injured tissue, neutrophils quickly phagocytize the parasites and it is now known that: they can remain infected for a while in the infection sites where they can even allow the multiplication of intracellular parasites. They can become apoptotic and later be phagocytized by macrophages or dendritic cells through the process of efferocytosis-the Trojan horse model (on top right side). They can transport parasites to the lymph nodes (on top left side). Taking into account the neutrophil's reverse transmigration mechanism: these cells could transport the parasites into the blood (3); once in the blood, they could be cleared in the liver (4) and/or the spleen (5); and, finally, infected senescent neutrophils in the blood, they could return to the bone marrow (6).

in a variety of host species, breeds and strains (human, canids and rodents like, hamsters and susceptible and resistant mice). Likewise, they were performed at different time intervals [48].

From the results, several chemotactic factors of neutrophils have been described showing components derived respectively from the insect vector, the parasite and the host.

From the vector insect, it was demonstrated that the phlebotomine saliva could induce an intense local neutrophil mobilization.

Regarding the components derived from the parasite, we have the promastigote secreting gel (PSG) and the chemotactic factor Leishmania (LCF) and, fi- 
nally, of the host factors described, there are several, IL-8, CXCL1, CXCL2, CXCL6 C3, IL-17 and GCP-2 and GCP-2, all extremely effective in chemotaxis of neutrophils [33].

The similarities of neutrophil response between Leishmania and other pathogens it is another important factor to be considered.

In Leishmania major, the CCR1 regulates both the leucocyte chemotaxis as well as the Th1/Th2 cytokine responses to leishmaniasis, besides it also enhances the susceptibility during early phase of infection. Coincidentally, in Ankara virus that may be carried by neutrophils from the skin to lymph nodes and bone marrow. CCR1 regulates both the leucocyte chemotaxis as well as the Th1/Th2 cytokine responses [49].

Correspondently, it was suggested that $L$. donovani induces defective antigen-specific CD8+ $\mathrm{T}$ cell responses in mice infected, in with it is produced CD8+ $\mathrm{T}$ cell responses with restricted clonal expansion.

In $L$. donovani infection, CD8+ T cells sustained only 8 - 9 cycles of division, instead of 19 , which would correspond to naive cells. In addition, they also did not accumulate in the spleen.

Even well throughout the infection, when considerable amounts of antigens are presented, CD8 $+\mathrm{T}$ cells undergo a second round of activation, but end up becoming dysfunctional and eventually die from "exhaustion". Similarly high antigen levels also have been described as a cause of CD8+T cell "exhaustion" during chronic viral infections [50].

In patients with active visceral leishmaniasis (L. infantum) the CD8+ T cells showed a reduced response after stimulation besides a higher percentage of apoptotic cells when compared to healthy controls. In the spleen of these patients, there was also a significant reduction in CD18 + CD45RO + CD8+ T cells, in contrast to CD18 + CD8+ T cells retained in bone marrow [50] [51].

Coincidentally, a compartmentalization of the response observed, was similar to what happens in viral infection when the antigen is firstly transported to the bone marrow [50].

Such compartmentalized response has already been described in dogs experimentally infected with $L$. infantum. Despite the parasite's cutaneous invasion, the local cytokine response was absent up to six months after the inoculum. It was suggested that the main target organs, like spleen and liver, showed a mixed cytokine immune response early on infection. On the other hand, the anti-inflammatory and immune responses in peripheral tissues were only activated posteriorly, during the chronic-patent stages of the disease [52].

There are many other indications that reinforce the possibility of reverse transmigrated neutrophils could have some role on the Leishmania dissemination in the host body. Significant numbers of neutrophils are present at the inoculation site, lesions, and draining lymph nodes from Leishmania-infected mice [53] and GM-CSF, delay the apoptosis of neutrophils by increasing mitochondrial stability and reducing caspase 3 activity [54].

Mice receiving intradermal parasites exhibited a greater influx of neutrophils 
to the site of infection, and these cells were better able to internalize promastigotes when compared to mice infected subcutaneously. This early neutrophilic response corresponded with a 10 -fold higher parasite burden in intra-dermally infected mice versus subcutaneously infected mice. It underscores the potential for distinct immunological outcomes when comparing infection in different tissue compartments [55].

In dogs experimentally infected with Leishmania infantum through intradermal inoculation of promastigotes, just 3 to $4 \mathrm{~h}$ after inoculation the parasites were observed inside of neutrophils and scarce macrophages.

Interestingly, those parasitized cells progressively disappeared from the skin and $24 \mathrm{~h}$ after inoculation parasites were no longer observed. It was suggested that the parasites could have been dispersed in the canine organism and the macrophages could have some role as "carriers", considering that later were observed parasites in the popliteal lymph node as well as a humoral response [56].

However, taking into account that the authors reported that the parasites were mainly within neutrophils, in addition to some macrophages, then it is perfectly plausible to assume that neutrophils could also be responsible for the spread of the parasites, at least in part.

In the ear dermis of supposedly resistant C57BL/6 mice infected with L. major or $L$. mexicana, it was observed that the parasites were initially in neutrophils and later in macrophages. Neutrophils represented more than $80 \%$ of the total number of infected cells in 12 to 24 hours, and each neutrophil presented at least one viable parasite. Actually, after in vitro infections $L$. major was able to survive within neutrophils for hours [33].

Once again, the only assumed form for the spread of the parasite was through macrophages, by phagocytosis of apoptotic neutrophils containing parasites (Trojan horse) or direct phagocytosis of parasites after their release by neutrophils. However, it is likely that neutrophils also play a role in clearance of the parasite through the mechanism of reverse transmigration.

In fact, a study involving the monitoring of BALB/mouse inflammatory cells, after intradermal inoculation of ears with $L$. infantum metacyclic promastigotes, showed that neutrophils contained the highest proportion of intracellular parasites 6 to $24 \mathrm{~h}$ after inoculation, in contrast, dermal macrophage harboring most intracellular parasites were observed only after 2 to 7 days [57].

Anyway, it is obvious that the role of neutrophils in the interaction with Leishmania composes a very complex puzzle, but from the description of the phenomenon of reverse transmigration of neutrophils as a common physiological phenomenon, in addition to the possibility of these cells transporting pathogens through the natural barriers of the host. This may suggest that the role of neutrophils in this interaction goes far beyond a simple Trojan horse.

\section{Conclusions}

Currently, in the life cycle of visceral leishmaniasis it has been assumed that ma- 
crophages infected are the most important cells regarding parasite dispersion in the mammal host body. On the other hand, neutrophils would serve mostly just as a Trojan horse, being responsible for silent transference of the parasite to macrophages. Actually, most of those articles presume that neutrophils are short-lived cells and the only way to promote the clearance of these cells from inflamed tissues is the mechanism of efferocytosis that is mainly achieved by the phagocytosis of apoptotic neutrophils by macrophages.

However, taking into account the description by Hughes J. et al. 1997 that, in an injured tissue, the emigration of neutrophils could exceed the clearance in situ by apoptosis.

In addition to the most recent evidence of the occurrence of this phenomenon called neutrophil, reverse transmigration was observed through several different animal models, and also, it was recognized as a very common physiological mechanism that can provide transport of microorganisms across the host's natural barriers.

We are suggesting that neutrophils may also play a role in the spread of the parasite from the skin to other tissues in mammalian hosts, especially in visceral leishmaniasis. Thus, the reverse transmigration of neutrophils could be responsible, at least in part, for the transport of the parasite to the main target organs, such as the liver, spleen and bone marrow.

\section{Acknowledgements}

We thank the IOC for the financial support for publishing the article.

\section{Author's Contributions}

Carreira JCA - conception, design, drafting and revising of the manuscript. Silva AVM-drafting the manuscript.

\section{Conflicts of Interest}

Authors declare they have no conflicts of interest.

\section{References}

[1] Leishmaniasis. https://www.who.int/health-topics/leishmaniasis

[2] Uzonna, J.E. and Liu, D. (2012) The Early Interaction of Leishmania with Macrophages and Dendritic Cells and Its Influence on the Host Immune Response. Frontiers in Cellular and Infection Microbiology, 2, 1-8. https://doi.org/10.3389/fcimb.2012.00083

[3] Bekkering, S. and Torensma, R. (2013) Another Look at the Life of a Neutrophil. World Journal of Hematology, 2, 44-58. https://doi.org/10.5315/wjh.v2.i2.44

[4] Xu, R., Huang, H., Zhang, Z. and Wang, F.S. (2014) The Role of Neutrophils in the Development of Liver Diseases. Cellular \& Molecular Immunology, 11, 224-231. https://doi.org/10.1038/cmi.2014.2

[5] Marie Lacy, H., Bowlin, A.K., Hennings, L., Scurlock, A.M., Nagarajan, U.M. and 
Rank, R.G. (2011) Essential Role for Neutrophils in Pathogenesis and Adaptive Immunity in Chlamydia caviae Ocular Infections. Infection and Immunity, 79, 1889-1897. https://doi.org/10.1128/IAI.01257-10

[6] Mortaz, E., Alipoor, S.D., Adcock, I.M., Mumby, S. and Koenderman, L. (2018) Update on Neutrophil Function in Severe Inflammation. Frontiers in Immunology, 9, 2171. https://doi.org/10.3389/fimmu.2018.02171

[7] Hyun, Y.M. and Hong, C.W. (2017) Deep Insight into Neutrophil Trafficking in Various Organs. Journal of Leukocyte Biology, 102, 617-629. https://doi.org/10.1189/jlb.1RU1216-521R

[8] Papayannopoulos, V. (2018) Neutrophil Extracellular Traps in Immunity and Disease. Nature Reviews Immunology, 18, 134-147. https://doi.org/10.1038/nri.2017.105

[9] Chatfield, S.M., Thieblemont, N. and Witko-Sarsat, V. (2018) Expanding Neutrophil Horizons: New Concepts in Inflammation. Journal of Innate Immunity, 10, 422-431. https://doi.org/10.1159/000493101

[10] Hind, L.E. and Huttenlocher, A. (2018) Neutrophil Reverse Migration and a Chemokinetic Resolution. Developmental Cell, 47, 404-405.

https://doi.org/10.1016/j.devcel.2018.11.004

[11] Buckley, C.D., Ross, E.A., McGettrick, H.M., Osborne, C.E., Haworth, O., Schmutz, C., Stone, P.C.W., Salmon, M., Matharu, N.M., Vohra, R.K., Nash, G.B. and Rainger, G. (2006) Identification of a Phenotypically and Functionally Distinct Population of Long-Lived Neutrophils in a Model of Reverse Endothelial Migration. Journal of Leukocyte Biology, 79, 303-311. https://doi.org/10.1189/jlb.0905496

[12] Hughes, J., Johnson, R.J., Mooney, A., Hugo, C., Gordon, K. and Savill, J. (1997) Neutrophil Fate in Experimental Glomerular Capillary Injury in the Rat: Emigration Exceeds In Situ Clearance by Apoptosis. American Journal of Pathology, 150, 223-234.

[13] Mathias, J.R., Perrin, B.J., Liu, T.X., Kanki, J., Look, A.T. and Huttenlocher, A. (2006) Resolution of Inflammation by Retrograde Chemotaxis of Neutrophils in Transgenic Zebrafish. Journal of Leukocyte Biology, 80, 1281-1288. https://doi.org/10.1189/jlb.0506346

[14] Woodfin, A., Voisin, M. B., Beyrau, M., Caille, D., Diapouli, F.M., Nash, G.B., Chavakis, T., Albeda, S.M., Rainger, G. Ed., Meda, P., Imhof, B.A. and Nourshargh, S. (2011) The Junctional Adhesion Molecule JAM-C Regulates Polarized Transendothelial Migration of Neutrophils in Vivo. Nature Immunology, 12, 761-769. https://doi.org/10.1038/ni.2062

[15] Wang, J., Hossain, M., Thanabalasuriar, A., Gunzer, M., Meininger, C. and Kubes, P. (2017) Visualizing the Function and Fate of Neutrophils in Sterile Injury and Repair. Science, 358, 111-116. https://doi.org/10.1126/science.aam9690

[16] Hirano, Y., Aziz, M. and Wang, P. (2016) Role of Reverse Transendothelial Migration of Neutrophils in Inflammation. Biological Chemistry, 397, 497-506. https://doi.org/10.1515/hsz-2015-0309

[17] Regli, I.B., Passelli, K., Hurrell, B.P. and Tacchini-Cottier, F. (2017) Survival Mechanisms Used by Some Leishmania Species to Escape Neutrophil Killing. Frontiers in Immunology, 8, 1558. https://doi.org/10.3389/fimmu.2017.01558

[18] Mirkovich, A.M., Galelli, A., Allison, A.C. and Modabber, F.Z. (1986) Increased Myelopoiesis during Leishmania major Infection in Mice: Generation of "Safe Targets", a Possible Way to Evade the Effector Immune Mechanism. Clinical Experimental Immunology, 64, 1-7. 
[19] Hosseini, R., Lamers, G.E.M., Soltani, H.M., Meijer, A.H., Spaink, H.P. and Schaaf, M.J.M. (2016) Efferocytosis and Extrusion of Leukocytes Determine the Progression of Early Mycobacterial Pathogenesis. Journal of Cell Science, 129, 3385-3395. https://doi.org/10.1242/jcs.135194

[20] Sant'Anna, M.R.V., Darby, A.C., Brazil, R.P., Montoya-Lerma, J. and Dillon, R.J. (2012) Investigation of the Bacterial Communities Associated with Females of Lutzomyia Sand Fly Species from South America. PLoS ONE, 7, e42531. https://doi.org/10.1371/journal.pone.0042531

[21] Moreland, J.G., Hook, J.S., Bailey, G., Ulland, T. and Nauseef, W.M. (2009) Francisella tularensis Directly Interacts with the Endothelium and Recruits Neutrophils with a Blunted Inflammatory Phenotype. American Journal of Physiology-Lung Cellular and Molecular Physiology, 296, L1076-L1084.

https://doi.org/10.1152/ajplung.90332.2008

[22] Dey, R., Joshi, A.B., Oliveira, F., Nakhasi, H.L., Valenzuela, J.G. and Kamhawi, S. (2018) Gut Microbes Egested during Bites of Infected Sand Flies Augment Severity of Leishmaniasis via Inflammasome-Derived IL-1b. Cell Host \& Microbe, 23, 134-143. https://doi.org/10.1016/j.chom.2017.12.002

[23] Nordenfelt, P. and Tapper, H. (2011) Phagosome Dynamics during Phagocytosis by Neutrophils. Journal of Leukocyte Biology, 90, 271-284.

https://doi.org/10.1189/jlb.0810457

[24] Kobayashi, S.D., Malachowa, N. and DeLeo, F.R. (2018) Neutrophils and Bacterial Immune Evasion. Journal of Innate Immunity, 1-10. https://doi.org/10.1159/000487756

[25] Spinner, J.L., Winfree, S., Starr, T., Shannon, J.G., Nair, V., Steele-Mortimer, O. and Hinnebusch, B.J. (2014) Yersinia pestis Survival and Replication within Human Neutrophil Phagosomes and Uptake of Infected Neutrophils by Macrophages. Journal of Leukocyte Biology, 95, 389-399. https://doi.org/10.1189/jlb.1112551

[26] Kobayashi, S.D., Malachowa, N. and DeLeo, F.R. (2017) Influence of Microbes on Neutrophil Life and Death, Frontiers in Cellular and Infection Microbiology, 7, 159. https://doi.org/10.3389/fcimb.2017.00159

[27] Kinkead, L.C., Fayram, D.C. and Allen, L.A.H. (2017) Francisella novicida Inhibits Spontaneous Apoptosis and Extends Human Neutrophil Lifespan. Journal of Leukocyte Biology, 102, 815-828. https://doi.org/10.1189/jlb.4MA0117-014R

[28] Köckritz-Blickwede, M.V., Blodkamp, S. and Nizet, V. (2016) Interaction of Bacterial Exotoxins with Neutrophil Extracellular Traps: Impact for the Infected Host. Frontiers in Microbiology, 7, 41. https://doi.org/10.3389/fmicb.2016.00402

[29] Juneau, R.A., Stevens, J.S., Apicella, M.A. and Criss, A.K. (2015) A Thermonuclease of Neisseria gonorrhoeae Enhances Bacterial Escape from Killing by Neutrophil Extracellular Traps. The Journal of Infectious Diseases, 212, 316-324. https://doi.org/10.1093/infdis/jiv031

[30] Beiter, K., Wartha, F., Albiger, B., Normak, S., Zychlinsky, A. and Henriques-Normark, B. (2006) An Endonuclease Allows Streptococcus pneumoniae to Escape from Neutrophil Extracellular Traps. Current Biology, 16, 401-407.

https://doi.org/10.1016/j.cub.2006.01.056

[31] Van Zandbergen, G., Klinger, M., Mueller, A., Dannenberg, S., Gebert, A., Solbach, W. and Laskay, T. (2004) Cutting Edge: Neutrophil Granulocyte Serves as a Vector for Leishmania Entry into Macrophages. The Journal of Immunology, 173, 6521-6525. https://doi.org/10.4049/jimmunol.173.11.6521

[32] Pitale, D.M., Gendalur, N.S., Descoteaux, A. and Shaha, C. (2019) Leishmania do- 
novani Induces Autophagy in Human Blood-Derived Neutrophils. The Journal of Immunology, 202, 1163-1175. https://doi.org/10.4049/jimmunol.1801053

[33] Hurrell, B.P., Regli, I.B. and Tacchini-Cottier, F. (2016) Different Leishmania Species Drive Distinct Neutrophil Functions. Trends in Parasitology, 32, 392-401. https://doi.org/10.1016/j.pt.2016.02.003

[34] Aga, E., Katschinski, D.M., Van Zandbergen, G., Laufs, H., Hansen, B., Muller, K., Solbach, W. and Laskay, T. (2002) Inhibition of the Spontaneous Apoptosis of Neutrophil Granulocytes by the Intracellular Parasite Leishmania major. The Journal of Immunology, 169, 898-905. https://doi.org/10.4049/jimmunol.169.2.898

[35] Davis, R.E., Thalhofer, C.J. and Wilson M.E. (2016) Infection and Activation of Human Neutrophils with Fluorescent Leishmania infantum. The Journal of Immunological Techniques \& Infectious Diseases, 5, 1-13.

https://doi.org/10.4172/2329-9541.1000146

[36] Rosales, C. (2018) Neutrophil: A Cell with Many Roles in Inflammation or Several Cell Types? Frontiers in Physiology, 9, 113. https://doi.org/10.3389/fphys.2018.00113

[37] Oualha, R., Barhoumi, M., Marzouki, S., Harigua-Souiai, E., Ahmed, M.B. and Guizani, I. (2019) Infection of Human Neutrophils with Leishmania infantum or Leishmania major Strains Triggers Activation and Differential Cytokines Release. Frontiers in Cellular and Infection Microbiology, 9, 153. https://doi.org/10.3389/fcimb.2019.00153

[38] Almeida, B.F.M., Narciso, L.G., Boscoa, A.M., Pereira, P.P., Braga, E.T., Avanço, S.V., Marcondes, M. and Ciarlini, P.C. (2013) Neutrophil Dysfunction Varies with the Stage of Canine Visceral Leishmaniosis. Veterinary Parasitology, 196, 6-12. https://doi.org/10.1016/j.vetpar.2013.02.016

[39] Karaji, N. and Sattentau, Q.J. (2017) Efferocytosis of Pathogen-Infected Cells. Frontiers in Immunology, 8, 1863. https://doi.org/10.3389/fimmu.2017.01863

[40] Rupp, J., Pfleiderer, L., Jugert, C., Moeller, S., Klinger, M., Dalhoff, K., Solbach, W., Stenger, S., Laskay, T. and van Zandbergen, G. (2009) Chlamydia pneumoniae Hides inside Apoptotic Neutrophils to Silently Infect and Propagate in Macrophages. PLoS ONE, 4, e6020. https://doi.org/10.1371/journal.pone.0006020

[41] Greenlee-Wacker, M.C., Rigby, K.M., Kobayashi, S.C., Porter, A.R., DeLeo, F.R. and Nauseef, W.M. (2014) Phagocytosis of Staphylococcus aureus by Human Neutrophils Prevents Macrophage Efferocytosis and Induces Programmed Necrosis. The Journal of Immunology, 192, 4709-4717. https://doi.org/10.4049/jimmunol.1302692

[42] Woodfin, A., Voisin, M.B., Beyrau, M., Colom, B., Caille, D., Diapouli, F.M., Nash, G.B., Chavakis, T., Albelda, S.M., Rainger, G.E., Meda, P., Imhof, B.A. and Nourshargh, S. (2012) Junctional Adhesion Molecule-C (JAM-C) Regulates Polarized Neutrophil Transendothelial Cell Migration in Vivo. Nature Immunology, 12, 761-769. https://doi.org/10.1038/ni.2062

[43] Oliveira, S., Rosowski, E.E. and Huttenlocher, A. (2016) Neutrophil Migration in Infection and Wound Repair: Going Forward in Reverse. Nature Reviews Immunology, 16, 378-391. https://doi.org/10.1038/nri.2016.49

[44] Duffy, D., Perrin, H.L., Abadie, V., Benhabiles, N., Boissonnas, A., Liard, C., Descours, B., Reboulleau, D., Bonduelle, O., Verrier, B., Rooijen, N.V., Combadie, C. and Combadie, B.H. (2012) Neutrophils Transport Antigen from the Dermis to the Bone Marrow, Initiating a Source of Memory CD8+ T Cells. Immunity, 37, 917-929. https://doi.org/10.1016/j.immuni.2012.07.015

[45] Coombesa, J.L., Charsara, B.A., Hana, S.J., Halkiasa, J., Chana, S.W., Koshyb, A.A., 
Striepend, B. and Robeya, E.A. (2013) Motile Invaded Neutrophils in the Small Intestine of Toxoplasma gondii-Infected Mice Reveal a Potential Mechanism for Parasite Spread. Proceedings of the National Academy of Sciences, 110, E1913-E1922. https://doi.org/10.1073/pnas.1220272110

[46] Ellett, F., Elks, P.M., Robertson, A.L., Ogryzko, N.V. and Renshaw, S.A. (2015) Defining the Phenotype of Neutrophils Following Reverse Migration in Zebrafish. Journal of Leukocyte Biology, 98, 975-981. https://doi.org/10.1189/jlb.3MA0315-105R

[47] Li, J.L.Y. and Ng, L.G. (2012) Peeking into the Secret Life of Neutrophils. Immunology Research, 53, 168-181. https://doi.org/10.1007/s12026-012-8292-8

[48] Ribeiro-Gomes, F.L. and Sacks, D. (2012) The Influence of Early Neutrophil-Leishmania Interactions on the Host Immune Response to Infection. Frontiers in Cellular and Infection Microbiology, 2, 59.

https://doi.org/10.3389/fcimb.2012.00059

[49] Rodriguez-Sosa, M., Rosas, L.E., Terrazas, L.I., Lu, B., Gerard, C. and Satoskar, A.R. (2003) CC Chemokine Receptor 1 Enhances Susceptibility to Leishmania major during Early Phase of Infection. Immunology Cell Biology, 2, 114-120. https://doi.org/10.1046/j.0818-9641.2002.01132.x

[50] Stäger, S. and Rafati, S. (2012) CD8+T Cells in Leishmania Infections: Friends or Foes? Frontiers in Immunology, 3, 5. https://doi.org/10.3389/fimmu.2012.00005

[51] Clarencio, J., Oliveira, C.I., Favali, C., Medina, O., Caldas, A., Costa, C.H., Costa, D.L., Brodskyn, C., Barral, A. and Barral-Netto, M. (2008) Could the Lower Frequency of CD8+CD18+CD45RO+ Lymphocytes be Biomarkers of Human VL? International Immunology, 21, 137-144. https://doi.org/10.1093/intimm/dxn131

[52] Rodríguez-Cortés, A., Carrillo, E., Martorell, S., Todolí, F., Ojeda, A., Martínez-Flórez, A., Urniza, A., Moreno, J. and Alberola, J. (2016) Compartmentalized Immune Response in Leishmaniasis: Changing Patterns throughout the Disease. PLOS ONE, 11, e0155224. https://doi.org/10.1371/journal.pone.0155224

[53] Prates, D.B., Araujo-Santos, T., Brodskyn, C., Barral-Neto, M., Barral, A. and Borges, V.M. (2012) New Insights on the Inflammatory Role of Lutzomyia longipalpis Saliva in Leishmaniasis. Journal of Parasitology Research, 2012, Article ID: 643029. https://doi.org/10.1155/2012/643029

[54] Witko-Sarsat, V., Rieu, P., Descamps-Latscha, B., Lesavre, P. and Halbwachs-Mecarelli, L. (2000) Neutrophils: Molecules, Functions and Pathophysiological Aspects. Laboratory Investigation, 80, 617-653. https://doi.org/10.1038/labinvest.3780067

[55] Carlsen, E.D., Liang, W., Shelite, T.R., Walker, D.H., Melby, P.C. and Soong, L. (2015) Permissive and Protective Roles for Neutrophils in Leishmaniasis. Clinical \& Experimental Immunology, 182, 109-118. https://doi.org/10.1111/cei.12674

[56] Santos-Gomes, G.M., Campino, L. and Abranches, P. (2000) Canine Experimental Infection: Intradermal Inoculation of Leishmania infantum Promastigotes. Memória do Instituto Oswaldo Cruz, 95, 193-198. https://doi.org/10.1590/S0074-02762000000200010

[57] Thalhofer, C.J., Chen, Y., Sudan, B., Love-Homan, L. and Wilson, M.E. (2011) Leukocytes Infiltrate the Skin and Draining Lymph Nodes in Response to the Protozoan Leishmania infantum chagasi. Infection and Immunity, 79, 108-117. https://doi.org/10.1128/IAI.00338-10 